\title{
Low temperature characterization of the photocurrent produced by two-photon transitions in a quantum dot intermediate band solar cell
}

\author{
E. Antolín , A. Martí , C.R. Stanley , C.D. Farmer , E. Cánovas , \\ N. López , P.G. Linares , A. Luque \\ Instituto de Energia Solar, Universidad Politécnica de Madrid, Ciudad Universitaria sn, 28040 Madrid, Spain \\ Department of Electronics and Electrical Engineering, University of Glasgow, Glasgow G12 8QQ, UK
}

\begin{abstract}
Conceived to exceed the conversion efficiency of conventional photovoltaic devices, the intermediate band solar cell bases its operation on exploiting, besides the usual band-to-band optical transitions, the absorption of two sub-bandgap photons. For the present, the only technology used to implement an intermediate band in real devices has been the growth of an InAs/GaAs quantum dot superlattice. In practice, the obtained material shows two limitations: the narrow energy gap between conduction and intermediate band and the appearance of growth defects due to the lattice stress. The consequences are the presence of non-radiative recombination mechanisms and the thermal escape of electrons from the intermediate to the conduction band, hindering the splitting of the quasi-Fermi levels associated with the intermediate and conduction bands and the observation of photocurrent associated with the two-photon absorption. By reducing the temperature at which the devices are characterised we have suppressed the parasitic thermal mechanisms and have succeeded in measuring the photocurrent caused by the absorption of two below bandgap photons. In this work, the characterization of this photocurrent at low temperature is presented and discussed.
\end{abstract}

Keywords: Intermediate band; Solar cells; Quantum dots

\section{Introduction}

Intermediate band solar cells (IBSC) base their operation on the use of intermediate band materials: semiconductors that possess a third energetic band, the intermediate band (IB), inbetween the bandgap formed by the conduction (CB) and valence (VB) bands (Fig. 1). As is the case for the $\mathrm{CB}$ and $\mathrm{VB}$ in conventional semiconductors, the IB must be separated from the other bands by a zero density of states, giving rise to two additional bandgaps, labeled $E_{\mathrm{L}}$ and $E_{\mathrm{H}}$ in the figure. Having three bandgaps, three optical transitions, labeled (1), (2) and (3), are caused by the absorption of photons of different energies. The IBSC theory also predicts that the electron-hole pairs produced can be extracted from the material at a voltage limited by the larger bandgap $E_{\mathrm{G}}$ and not by the smaller ones. To achieve this, the IB material is sandwiched between two layers of conventional semiconductor (emitters) of opposite doping, which provide the required selective contacts to the $\mathrm{CB}$ and the VB, and leave the IB unconnected with the external contacts. Fig. 2 represents the band diagram of the IBSC in operation, showing the three quasi-Fermi levels that describe the electronic population in the IB material. One of them is pinned to the IB, which means that this band is half-occupied and thus possesses a steady electronic population. This implies that the double-absorption process (sum of absorptions (1) and (2)) that confers extra electron-hole pairs to the IBSC is not given necessarily by the improbable simultaneous absorption of two photons by one VB electron.

Thanks to the IB, the cell is able to deliver more current than a single-gap solar cell of the same bandgap $E_{G}$, while preserving its voltage. This confers to the novel concept an efficiency limit 


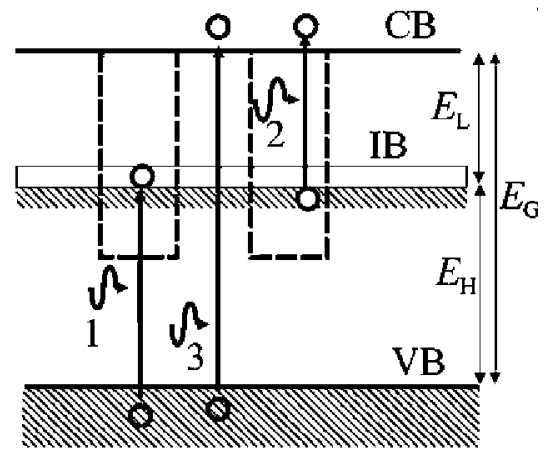

Fig. 1. Equilibrium band diagram and possible absorption processes of an IB material. The CB offsets induced by the QDs are also shown.

of $63.2 \%$ under maximum concentration, the same value as for a series connected triple tandem cell and to be compared with the Shockley-Queisser limit of $40.7 \%$ for conventional solar cells . This limit could be raised to over $70 \%$ by combining two IBSCs in a series tandem

For the moment, the only approach that has allowed the implementation of practical IBSCs is based on the use of quantum dot (QD) superlattices The quantum confining potential of a QD is three dimensional. Therefore it produces atom-like discrete levels and their electronic wavefunctions may overlap with those of levels in neighbour QDs if they are close enough. Given an appropriate band offset between the dot and barrier materials, these discrete levels can produce an isolated IB, as it is shown schematically in Fig. 1. Following this approach, IBSCs have been fabricated using QDs of InAs in

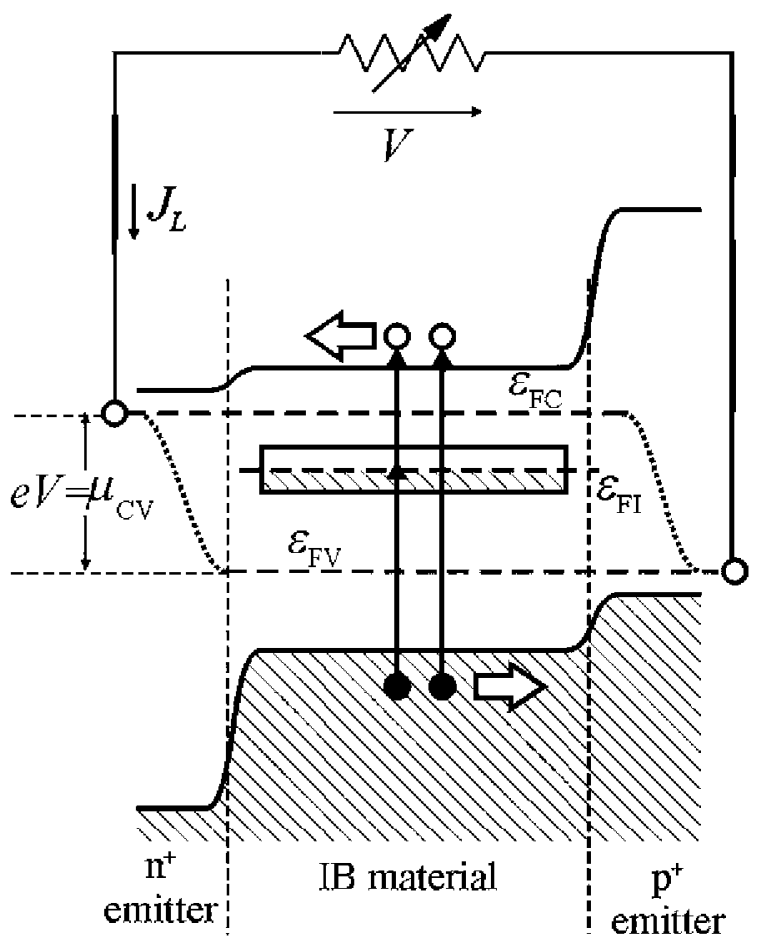

Fig. 2. Band diagram and quasi-Fermi levels of an IBSC in working conditions,
GaAs and some experimental knowledge has already been gained about them The use of QDs has some drawbacks, such as the low QD density and number of layers achievable, together with the weak absorption between IB and CB. Nevertheless, it is nowadays a quite mature technology and has proven a useful approach to test the IBSC principles of operation.

An advantage of the QD approach is that QDs exhibit a phonon-bottleneck effect: as a consequence of their big volume relative to the lattice spacing and hence small volume in reciprocal space, they do not possess large vibrational modes that can be used to thermalize carriers between bands Unfortunately, it has been observed that during the growth of real QD material, disorder defects and dislocations appear due to inelastic relaxation of the strain in the lattice Furthermore, it has been suggested that real QD material exhibit a proper phonon-bottleneck effect only for low temperatures

This fact, together with the low absorption exhibited by the QDs in the IB-CB transition, has made impossible until now the observation of photocurrent produced by absorption of two subbandgap photons through the IB at room temperature. However, we have succeeded in measuring this photocurrent by lowering the working temperature of the device, corroborating one of the key principles of the IBSC concept In this work, further experiments have been carried out to characterise this photocurrent.

\section{The QD-IBSC samples}

The QD-IBSCs used in our experiments were processed from InAs/GaAs QD structures grown by molecular beam epitaxy using the Stranski-Krastanov growth mode [16]. Fig. 3 shows the internal structure of the devices, which have a junction area of $4 \mathrm{~mm}^{2}$. The In flux was calibrated by reflection high energy electron diffraction (RHEED) intensity oscillations from an InAs wafer and set to a growth rate of 0.032 monolayers per second $(\mathrm{ML} / \mathrm{s})$. A stack of 10 QDs and capping layers was grown, each one consisting of $3.2 \mathrm{ML}$ of InAs deposited at $460^{\circ} \mathrm{C}$ followed by $5 \mathrm{~nm}$ of un-GaAs, Si-doping with an areal density of $1 \times 10^{10} \mathrm{~cm}^{-2}$ and finally $3.5 \mathrm{~nm}$ of un-GaAs to complete the capping of the dots. The density of the modulated

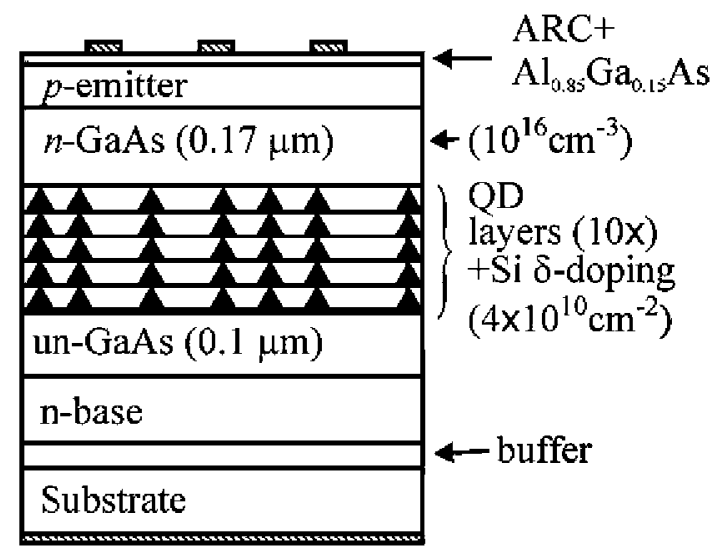

Fig. 3. Sketch of the structure of the QD-IBSC samples used in the experiments, 
$\mathrm{Si} \delta$-doping equals that of the dots, since its purpose is to induce the required half-filling of the IB At this point in the growth of the stack, the substrate temperature was raised to $580^{\circ} \mathrm{C}$ and held there for $10 \mathrm{~min}$ to smooth the surface of the GaAs cap The wafer temperature was then lowered back to $460^{\circ} \mathrm{C}$ and the next period of the QD stack grown.

The samples exhibit at room temperature a quantum efficiency as depicted in Fig. 4. The quantum efficiency of a GaAs reference cell is also shown for comparison. The structure of this reference cell is identical to that of the QD-IBSC (Fig. 3), with the only difference of not containing the QD layers. It can be observed that for wavelengths greater than $879 \mathrm{~nm}$, that corresponds to the gap energy of GaAs $(1.41 \mathrm{eV})$, the response of the reference cell decays abruptly, while that of the QD-IBSC sample shows further photocurrent until approx. $1300 \mathrm{~nm}$ and then decays. This extra photocurrent is caused by the absorption of photons from the VB to the IB. The most probable reasons for this sub-bandgap photocurrent to be collected at high temperatures are mechanisms of thermal and tunneling escape of carriers form the IB to the CB. From this data it is deduced that the two extra bandgaps of our QD material are $E_{\mathrm{H}} \approx 0.95 \mathrm{eV}$ and $E_{\mathrm{L}} \approx 0.46 \mathrm{eV}$, although given the narrowing of the fundamental bandgap due the presence of the dots, $E_{\mathrm{L}}$ might have a much lower value $(\sim 0.3 \mathrm{eV})$

\section{Experimental}

The experimental set-up to measure the photocurrent produced by absorption of two sub-bandgap photons is represented in Fig. 5. The QD-IBSC sample is placed in a closed-cycle He cryostat in which the temperature of the device can be lowered down to $6 \mathrm{~K}$. The sample faces two windows of the cryostat that are used to illuminate it simultaneously with two different light sources. The primary light beam, originated by a halogen lamp, is tuned from the UV to the IR wavelengths by a monochromator as in a conventional spectral response measurement. Its objective is to pump electrons from the VB to the IB. The

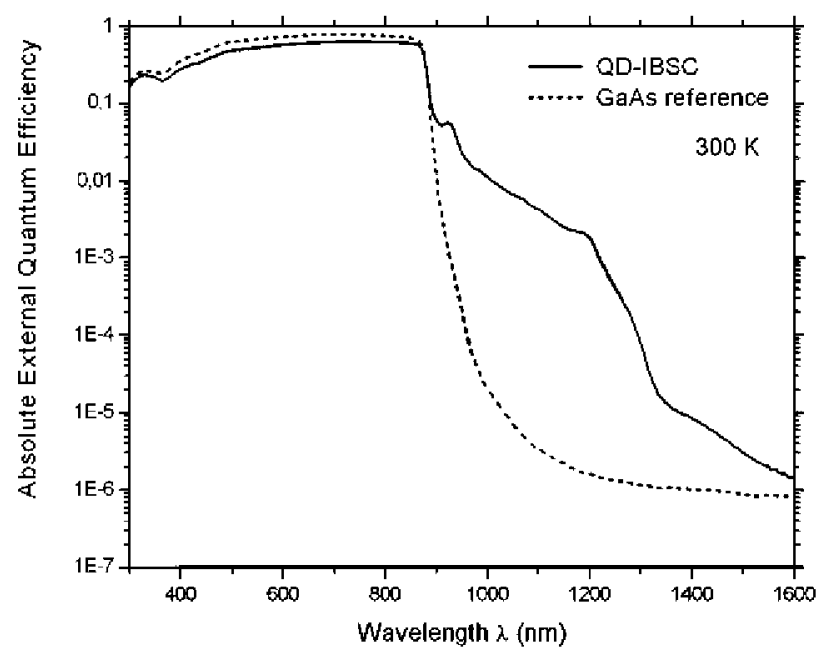

Fig. 4. Absolute external quantum efficiency of the QD-IBSC samples (solid line). The quantum efficiency of a GaAs cell with identical structure but no QD material is also shown for reference (dashed line).

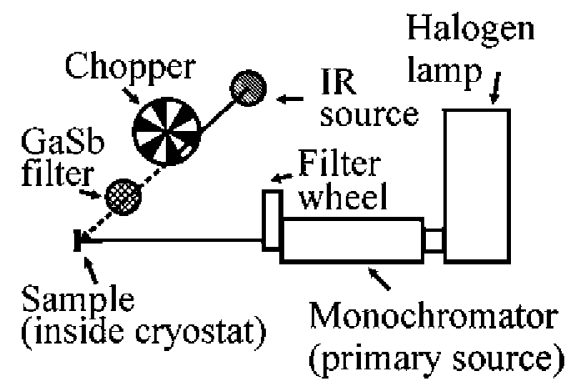

Fig. 5. Experimental set-up developed for measurement of photocurrent due to absorption of two sub-bandgap photons

secondary light comes from an infrared source (filament heated) and is filtered by a $350 \mu \mathrm{m}$ thick GaSb wafer. The filter assures that no photons from the secondary source with energy higher than $0.7 \mathrm{eV}$ can reach the cell and thus, according to the $\mathrm{QE}$ of the samples, this source can only cause promotions from the IB to the CB.

To ensure the distinction between both contributions and the specific record of two-photon photocurrent, a frequency modulated technique has been used. The secondary beam has been optically chopped at a frequency of $13 \mathrm{~Hz}$ and the cell has been connected to a low noise transresistance pre-amplifier. The pre-amplifier biases the QD-IBSC at $0 \mathrm{~V}$, which means that in all the presented measurements the cell is working in shortcircuit. This is a very relevant point, because it means that the signal recorded has a photovoltaic origin and is not related to an optically induced conductivity change in the IB material. The continuous light from the primary source gives rise to a dc current in the cell that is filtered in the pre-amplifier. Then, the ac amplified signal, transformed to a voltage signal, is delivered to a digital lock-in amplifier synchronized to the chopper of the secondary beam. The signal that is measured in the lock-in is then, effectively, a double-absorption photocurrent: it results from the electron-hole pairs created in response to the excitation between IB and $\mathrm{CB}$ by the secondary light, when a pumping level between VB and IB is provided by the primary light. Given the small magnitude of the expected signal and the fact that the dc current caused by the primary beam may be orders of magnitude higher, low noise measurement techniques, such as wire screening, adequate groundings and a previous noise spectral record, are required in the set-up.

\section{Results}

Fig. 6 shows the dependence on the temperature of the double-absorption photocurrent for a fixed wavelength of the primary source $(790 \mathrm{~nm})$. It can be seen that at high temperatures only electrical noise is recorded by the system. When the temperature lowers to about $90 \mathrm{~K}$ a signal of photocurrent emerges from the noise and it rises as the temperature continues decreasing until it reaches the value of $4.510^{-11} \mathrm{~A} / \mathrm{mm}^{2}$. We chose two temperatures: $80 \mathrm{~K}$, which is close to the turning point of the double-absorption photocurrent, and $6 \mathrm{~K}$, and perform a spectral scan of the photocurrent by tuning the wavelength of the primary source. The results are presented in Fig. 7 with the labels (b) and (c), respectively. We have added to the 


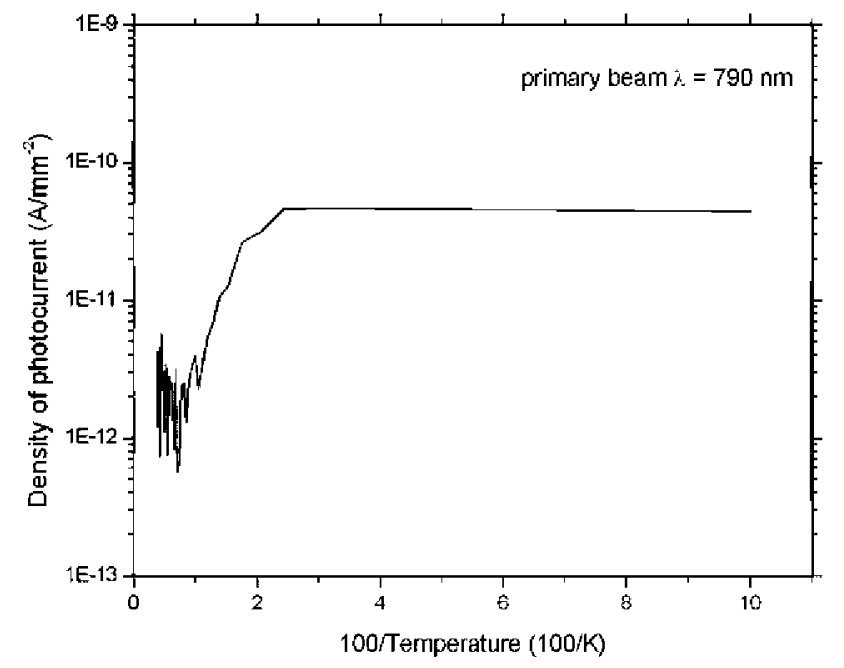

Fig. 6. Dependence of the photocurrent due to double sub-bandgap absorption on the temperature of the QD-IBSC.

plot the curve (a), the photocurrent produced by illuminating the QD-IBSC only with the primary source and the curve (d), the noise level of the experiment, both recorded at $80 \mathrm{~K}$.

Curve (a) has been obtained by chopping the primary light beam and disconnecting the secondary source and its chopper. It equals the dc current flowing through the device when the double-absorption experiment is performed (divided by a factor of $2^{1 / 2}$, because in this case it is recorded as ac signal and thus the magnitude measured is the rms value). It must be noticed that this current is for a wide range of wavelengths three orders of magnitude higher than the relevant photocurrent. Besides, it can be observed that the signal produced by sub-bandgap photons, promoting electrons only from the VB to the IB, does not disappear at $80 \mathrm{~K}$. This is also the case for lower temperatures, which indicates that probably carriers are escaping

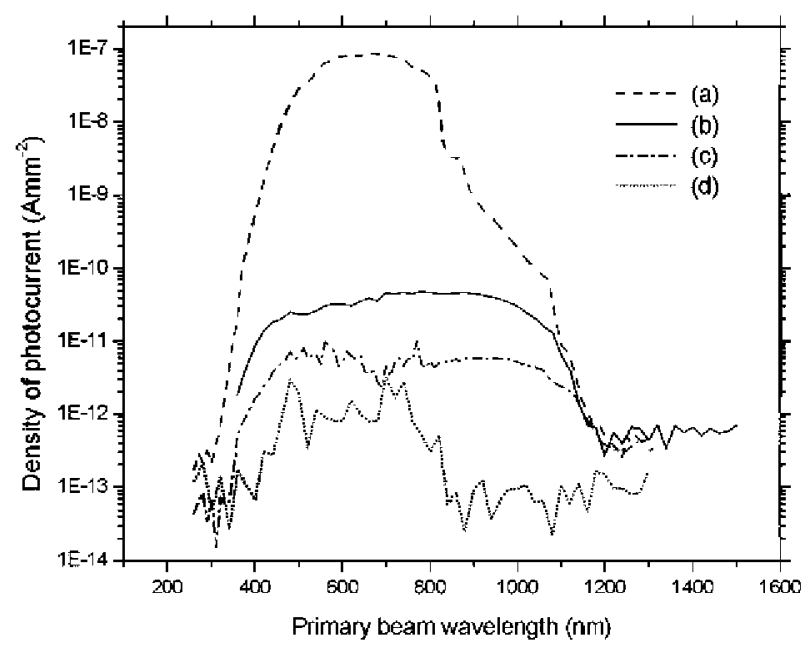

Fig. 7. Measurements of the QD-IBSC photocurrent versus the wavelength of the primary light: (a) photocurrent produced by the primary light alone at $80 \mathrm{~K}$, (b) photocurrent measured when the secondary light is on and chopped at $6 \mathrm{~K}$, (c) same measurement at $80 \mathrm{~K}$, and (d) result at $80 \mathrm{~K}$ of the same double-photon photocurrent measurement, but letting the secondary source off and chopped (represents the noise level of the experiment). from the dots by tunnel effect This is justified by the fact that at zero bias the QDs lie in the space charge region of the junction, where the bands are not flat. It must be noticed that the $\mathrm{n}$-doped and intrinsic layers that surround the QD material have been designed to flatten the bands under voltage bias and in all this experiments the QD-IBSC is short-circuited.

Curve (d) is the result of exactly the same measurement as for curve (c), where the secondary source is the one chopped, but in this case this source is turned off. Hence, curve (d) represents the noise level of the experiment at $80 \mathrm{~K}$. It can be observed that it is not constant for all wavelengths of the primary source. In the range at which the absorption of primary light is very high (between 450 and $850 \mathrm{~nm}$ ) the noise level raises. Curves (b) and (c) show that the photocurrent due to electron-hole pairs created by double absorptions grows when decreasing the temperature, in concordance with Fig. 6. At $80 \mathrm{~K}$ the current measured lies still very close to the noise in the system, while for lower temperatures its magnitude has raised enough to be clearly recorded.

Fig. 8 shows the measurement of photocurrent due to twophoton absorption at different low temperatures $(40 \mathrm{~K}, 20 \mathrm{~K}$ and $6 \mathrm{~K}$ ). Since the signal measured is in fact very low, it is necessary to check that it actually comes from a double absorption and excludes the possibility that, in spite of the GaSb filter used, spurious photons of too high energy may reach the cell from the chopped secondary source. This possibility is discarded by the dependence of the photocurrent on the primary light wavelength. The signal arises when primary photons impinging the cell achieve a significant amount (this cut-on is given by the optics and the secondary filters used in the monochromator) and decays when the absorption of primary photons is too low. Moreover, the signal does not decay abruptly when the bandgap of GaAs is reached, excluding the contribution to the photocurrent of primary light reflected on the chopper of the secondary source. On the contrary, the photocurrent collected is higher when the primary light wavelength is over approx. $700 \mathrm{~nm}$. When the energy of the primary photons

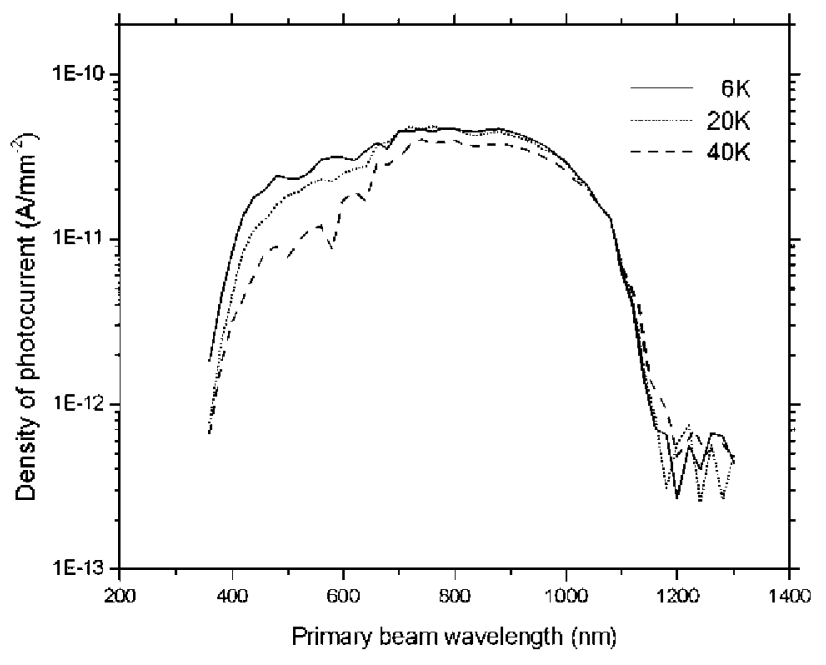

Fig. 8. Measurements of the QD-IBSC photocurrent due to double sub-bandgap absorption versus the wavelength of the primary light for different temperatures of the device. 
exceeds that of the bandgap of the cell, they contribute to the double photon transitions by promoting an electron from the deeper bands of the VB to the IB. Nevertheless, these energetic photons have a high probability of being absorbed through VBto-CB transitions in the emitter and not reaching the QD material, which could explain the form of the curves. This does not mean that the photocurrent measured has to drop completely for high energy photons that are very efficiently absorbed in the emitter, since it is expected that the emitter re-radiates a part of the light absorbed to the inner of the cell where the QD material is located, especially at low temperatures. It is also remarkable that at the temperature of $20 \mathrm{~K}$ the double-absorption photocurrent has already reached its maximum value for primary photons of wavelength over $700 \mathrm{~nm}$.

\section{Conclusions}

The photocurrent produced by double-absorption of subbandgap photons predicted by the IBSC model has been measured in QD-IBSCs fabricated with InAs/GaAs material using a modulated technique with two light beams. The photocurrent was not measurable for temperatures higher than $90 \mathrm{~K}$ and its value increased for lower temperatures, showing a smaller increase from $40 \mathrm{~K}$ to about $20 \mathrm{~K}$, where it achieves its maximum value. The dependence of this photocurrent on the energy of photons producing transitions from the VB to IB has been measured for different temperatures, showing that when the energy exceeds approx. $1.7 \mathrm{eV}$ the photocurrent decreases, probably due to absorption in the emitter.

\section{Acknowledgments}

This work has been supported by the project FULLSPECTRUM, funded by the European Commission under Contract No. SES6-CT-2003-502620, the Spanish Plan for R\&D ConsoliderGENESIS FV (CSD2006-00004) and the Comunidad de Madrid Program NUMANCIA (S-0505/ENE/000310). E.A. acknowledges the UPM and E.C. acknowledges the Spanish Science and
Technology Ministry for their financial support through their grant programs.

\section{References}

A. Luque, A. Martí, Phys. Rev. Lett. 78 (1997) 5014.

A. Luque, A. Martí, Prog. Photovolt: Res. Appl. 9 (2001) 73.

A.S. Brown, M.A. Green, Phys. E 14 (2002) 96.

W. Shockley, H.J. Queisser, J. Appl. Phys. 32 (1961) 510.

E. Antolín, A. Martí, A. Luque, Proc. of the 21st European Photovoltaic Solar Energy Conference, Munich: WIPRenewable Energies and ETA, Dresden, Germany, 2006, p. 412.

A. Marti, L. Cuadra, A. Luque, Proc. of the 28th IEEE Photovoltaics Specialists Conference, IEEE, New York, 2000.

A. Luque, A. Martí, N. López, E. Antolín, E. Cánovas, C.R. Stanley, C. Farmer, P. Díaz, J. Appl. Phys. 99 (2006).

A. Luque, A. Martí, N. López, E. Antolín, E. Cánovas, C. Stanley, C. Farmer, L.J. Caballero, L. Cuadra, J.L. Balenzategui, Appl. Phys. Lett 87 (2005)

A. Martí, N. López, E. Antolín, E. Cánovas, C. Stanley, C. Farmer, L. Cuadra, A. Luque, Thin Solid Films 511-512 (2006) 638.

J. Urayama, T.B. Norris, J. Singh, P. Bhattacharya, Phys. Rev. Lett. 86 (2001) 4930.

J.S. Wang, J.F. Chen, J.L. Huang, P.Y. Wang, X.J. Guo, Appl. Phys. Lett. 77 (2000) 3027 .

S.W. Lin, C. Balocco, M. Missous, A.R. Peaker, A.M. Song, Phys. Rev. B 72 (2005) 165302 (1.

F. Quochi, M. Dinu, J. Shah, L.N. Pfeiffer, K.W. West, P.M. Platzman, Phys. Rev. B (2002) 161308.

J. Urayama, T.B. Norris, H. Jiang, J. Singh, P. Bhattacharya, Appl. Phys. Lett. 80 (2002) 2162

A. Marti, E. Antolin, C.R. Stanley, C.D. Farmer, N. Lopez, P. Diaz, E. Canovas, P.G. Linares, A. Luque, Phys. Rev. Lett. 97 (2006) 247701.

M. Sugawara, Self-assembled InGaAs/GaAs Quantum Dots, Academic Press, 1999.

A. Martí, L. Cuadra, A. Luque, IEEE Trans. Electron Devices 48 (2001) 2394.

E.C.L. Ru, A.J. Bennett, C. Roberts, R. Murray, J. Appl. Phys. 91 (2002) 1365.

E.C.L. Ru, P. Howe, T.S. Jones, Phys. Rev. B 67 (2003).

A. Martí, C.R. Stanley, A. Luque, in: T. Soga (Ed.), Nanostructured Materials for Solar Energy Conversion, Elsevier, 2006, Chap 17.

P.W. Fry, I.E. Itskevich, S.R. Parnell, J.J. Finley, L.R. Wilson, K.L. Schumacher, D.J. Mowbray, M.S. Skolnick, M. Al-Khafaji, A.G. Cullis, M. Hopkinson, J.C. Clark, G. Hill, Phys. Rev. B 62 (2000) 16784. 